\title{
Patient-provider Communication in Cancer Screening
}

G eneral internists frequently offer screening tests for cancer to their patients. Shared decision making, in which the patient and the physician discuss and make decisions together, is necessary for screening to have maximum impact with minimum cost. Only then can the physician understand the patient's overall priorities, decision-making behaviors, and comprehension of the risk of developing the cancer and the benefits and risks of the test (and any follow-up interventions). Although physicians are guided by scientific evidence, patients want care that addresses their largely culturally determined personal values. ${ }^{1}$ Patient-physician communication becomes even more important when the patient and the physician come from different cultures. In this issue of JGIM, 3 articles illustrate the importance of different aspects of patient-physician communication to cancer screening.

Screening for prostate cancer with the prostatespecific antigen (PSA) test is a controversial practice, in part because of the lack of evidence that it reduces mortality. In situations such as this, shared decision making is of paramount importance. ${ }^{2}$ Chan et al. surveyed 450 internists, family practitioners, and urologists about 17 key facts to discuss with men age $\geq 50$ regarding PSA screening. ${ }^{3}$ Interestingly, although most supported such screening, urologists and nonurologists disagreed about the importance of informing their patients about 9 facts. Nonurologists were more likely than urologists to rate facts reflecting uncertainty, e.g., statements about the PSA controversy, prostate cancer risk, and treatment efficacy, as highly important for patients to know. Clearly, specialty training biases how physicians communicate information to patients. The study also illustrates the difficulties of shared decision making. Confronted with 17 different "facts" about a controversial screening test, patients may make decisions based not on the information provided but simply on their impression of the test or their physician's recommendation. Decision aids may facilitate shared decision making in office practice. ${ }^{4}$ Further research is needed to learn how much and what kind of information is helpful to patients in uncertain situations. ${ }^{5}$ In addition, for ethnic minority patients, particularly those who do not speak English primarily and those from other cultures, physicians need to learn how to break through language and literacy barriers, ${ }^{6}$ how to transmit concepts of probability and uncertainty, ${ }^{7,8}$ and how to employ a familial, rather than an individual, model of decision making. ${ }^{9,10}$
In contrast, screening for cervical cancer with the Pap test has been so successful in reducing mortality that recent guidelines recommend decreasing its frequency among low-risk women. However, ethnic disparities in Pap test screening remain, particularly among Asian-American women. ${ }^{11-14}$ Chaudry et al. report that only $73 \%$ of 225 South-Asian women in the United States who responded to a national mailed survey had had a Pap test within 3 years. ${ }^{15}$ Given that the survey was conducted only in English and had a limited response rate, the actual rate of Pap testing among all South-Asian women may be even lower. In addition, all women, but ethnic minority women in particular, tend to over-report their Pap test receipt. ${ }^{16}$ Socioeconomic and access barriers may account for low Pap screening rates in some ethnic minority women, but South-Asian women, despite having higher income levels and insurance, were less likely to have Pap tests compared to respondents to the 1999 National Health Interview Survey. Asian women may not obtain screening due to cultural factors such as a lack of a preventive care orientation or personal modesty. ${ }^{6,17}$ For others, physician recommendation and patient request may be the most important determinants of Pap test receipt. ${ }^{18}$ If physicians learn how to recommend the test in a culturally appropriate manner, patients may be able to overcome their reluctance. ${ }^{6}$

Finally, Turner et al. remind us that good patientphysician communication is important to the follow-up of screening tests, which are effective only if abnormal results are evaluated appropriately and promptly. ${ }^{19}$ This study evaluated the ordering of complete diagnostic evaluation (CDE) of the colon (colonoscopy or barium enema and flexible sigmoidoscopy) for patients with positive fecal occult blood tests (FOBTs) in primary care practices in a large HMO. Physicians ordered CDE within 180 days for only $69.5 \%$ of the positive-FOBT patients. Women were significantly less likely to have had CDE, perhaps due to the perception that their colon cancer risk was lower. Not surprisingly, patients whose physicians reported low intention to order CDE were significantly less likely to have had one. Some physicians may be unwilling to order uncomfortable, costly, and potentially risky follow-up tests to evaluate a positive FOBT, a screening test with low positive predictive value. If so, these issues should certainly be discussed with patients before ordering FOBT. In this case, effective patient-physician communication requires discussion not only of the different types of colon cancer screening tests, but also the patient's 
risk for developing colon cancer, the likelihood of a positive or a negative result, and the implications of follow-up procedures. System improvements to ensure adequate follow-up of abnormal screening tests may enhance overall patient-physician communication ${ }^{20,21}$ and also, in particular, benefit ethnic minorities, who have lower rates of follow-up ${ }^{22}$ and more difficulty negotiating the complex health care system. ${ }^{6}$

Good communication with patients about screening tests takes time, particularly when the patients are from another culture or speak another language. Yet, it is during these discussions that physicians come to understand their patients' cultures, values, and priorities. Where did they come from? How and how long do they want to live? What kind of medical care do they want? How much uncertainty can they live with? How do they make decisions? Besides the early detection of cancer, this understanding is the reward of shared decision making in cancer screening. - TuNG T. NGUYEN, MD, STEPHEN J. MCPHEE, MD, Vietnamese Community Health Promotion Project, Division of General Internal Medicine, Department of Medicine, University of California-San Francisco, San Francisco, Calif.

\section{REFERENCES}

1. Kleinman A, Eisenberg L, Good B. Culture, illness and care: clinical lessons from anthropologic and cross-cultural research. Ann Intern Med. 1978;88:251-8.

2. Walsh JME, McPhee SJ. Prevention in the year 2002: some news, some issues. Prim Care. 2002;29:727-49.

3. Chan EYC, Vernon SW, Haynes MC, O'Donnell FT, Ahn C. Physician perspectives on the importance of facts men ought to know about prostate specific antigen testing. $\mathrm{J}$ Gen Intern Med. 2003; 18:350-6.

4. Barry MJ. Health decision aids to facilitate shared decision making in office practice. Ann Intern Med. 2002;136:127-35.

5. Edwards A, Elwyn G. How should effectiveness of risk communication to aid patients' decisions be judged? A review of the literature. Med Decis Making. 1999;19:428-34.

6. McPhee SJ. Caring for a 70-year-old Vietnamese woman. JAMA. 2002;287:495-504.
7. Bogardus ST Jr, Holmboe E, Jekel JF. Perils, pitfalls, and possibilities in talking about medical risk. JAMA. 1999; 281:1037-41.

8. Wolf AM, Becker DM. Cancer screening and informed patient discussions. Truth and consequences. Arch Intern Med. 1996; 156: 1069-72.

9. Blackhall LJ, Murphy ST, Frank G, Michel V, Azen S. Ethnicity and attitudes toward patient autonomy. JAMA. 1995;274:820-5.

10. Kagawa-Singer M, Blackhall LJ. Negotiating cross-cultural issues at the end of life. JAMA. 2001;286:2993-3001.

11. Jenkins CNH, McPhee SJ, Bird JA, et al. Effect of a media-led education campaign on breast and cervical cancer screening among Vietnamese American women. Prev Med. 1999;28:395-406.

12. Kagawa-Singer M, Pourat N. Asian American and Pacific Islander breast and cervical carcinoma screening rates and Healthy People 2000 objectives. Cancer. 2000;89:696-705.

13. Maxwell AE, Bastani R, Warda US. Demographic predictors of cancer screening among Filipino and Korean immigrants in the United States. Am J Prev Med. 2000;18:62-8.

14. Juon HS, Choi Y, Kim MT. Cancer screening behaviors among Korean-American women. Cancer Detect Prev. 2000;24:589-601.

15. Chaudry S, Fink A, Gelberg L, Brook R. Utilization of Papanicolaou smears by South Asian women living in the United States. J Gen Intern Med. 2003;18:377-84.

16. McPhee SJ, Nguyen TT, Shema SJ, et al. Validation of recall of breast and cervical cancer screening by women in an ethnically diverse population. Prev Med. 2002;35:463-73.

17. Yi J. Factors associated with cervical cancer screening behavior among Vietnamese women. J Community Health. 1994;19: 189-200.

18. Nguyen TT, McPhee SJ, Nguyen T, Lam T, Mock J. Predictors of cervical Pap smear screening recognition, intention, and receipt among Vietnamese-American women. Am J Prev Med. 2002;23: 207-14.

19. Turner B, Myers RE, Hyslop T, et al. Physician and patient factors associated with ordering a colon evaluation after a positive fecal occult blood test. J Gen Intern Med. 2003;18:357-63.

20. Burack RC, Simon MS, Stano M, George J, Coombs J. Follow-up among women with an abnormal mammogram in an HMO: is it complete, timely, and efficient? Am J Manag Care. 2000;6: 1102-13.

21. Kerlikowske K. Timeliness of follow-up after abnormal screening mammography. Breast Cancer Res Treat. 1996;40:53-64.

22. Chang SW, Kerlikowske K, Napoles-Springer A, Posner SF, Sickles EA, Perez-Stable EJ. Racial differences in timeliness of follow-up after abnormal screening mammography. Cancer. 1996;78: $1395-402$. 